\title{
Circuit
}

Musiques contemporaines

\section{Lire Xenakis}

À propos du recueil d'écrits de Xenakis Kéleüta

On reading Xenakis

The essays in Kéleuita

\section{Serge Provost}

Volume 5, numéro 2, 1994

Espace Xenakis

URI : https://id.erudit.org/iderudit/902108ar

DOI : https://doi.org/10.7202/902108ar

Aller au sommaire du numéro

Éditeur(s)

Les Presses de l'Université de Montréal

ISSN

1183-1693 (imprimé)

1488-9692 (numérique)

Découvrir la revue

Citer cet article

Provost, S. (1994). Lire Xenakis : à propos du recueil d'écrits de Xenakis Kéleüta. Circuit, 5(2), 69-75. https://doi.org/10.7202/902108ar

\section{Résumé de l'article}

Ces écrits échelonnés de 1955 à 1988 et réunis sous le titre Kéleüta, donnent un excellent aperçu de l'ampleur de la pensée de leur auteur. Serge Provost a tâché d'en définir les traits essentiels et, partant, de mettre en perspective l'importance de la contribution de Xenakis au développement de la création musicale et de la pensée artistique en général.
Tous droits réservés @ Les Presses de l’Université de Montréal, 1994
Ce document est protégé par la loi sur le droit d'auteur. L’utilisation des services d’Érudit (y compris la reproduction) est assujettie à sa politique d'utilisation que vous pouvez consulter en ligne.

https://apropos.erudit.org/fr/usagers/politique-dutilisation/ 


\title{
Lire Xenakis
}

\author{
À propos du recueil d'écrits de \\ Xenakis : Kéleüta ${ }^{(1)}$ \\ Serge Provost
}

Les écrits des compositeurs sont précieux. Non seulement nous éclairentils sur leur œuvre musicale (surtout au vingtième siècle où les langages musicaux ne s'insèrent plus nécessairement dans une pratique communel, mais surtout ils permettent la communication de concepts plus généraux, théoriques, scientifiques et philosophiques. Ce qui a pour avantage d'insérer la réflexion des créateurs dans les courants de pensée d'une époque et de placer le débat entourant les considérations conceptuelles d'un art, abstrait par définition, sur le plan plus général des idées. En outre, cela peut et doit avoir pour conséquence de restaurer l'importance de la musique comme élément essentiel de l'évolution de la culture et de la pensée en général ${ }^{(2)}$.

De ce point de vue, les textes de Xenakis, rassemblés dans ce recueil, sont particulièrement pertinents. Citons la préface de Benoît Gibson :

Si les termes en lesquels il s'exprime sur la musique ont un support formalisé, c'est pour en étendre l'application et l'accès : l'universalité des mathématiques se substitue aux acquis d'une tradition, et la musique s'insère dans un champ plus vaste, car elle profite des développements techniques et conceptuels d'autres domaines de la pensée, tout en gardant sa propre autonomie. En ce sens l'apport de Xenakis n'est pas tant l'élaboration ou le développement d'une technique qu'une réflexion globale sur les enjeux de la création. Solitaire, mais précurseur, il a toujours porté au-devant de son temps la nécessite d'en élargir le champ et l'environnement. (1994, p. 11 .)

Les quinze textes dont il sera question ici ont été écrits sur plus d'une trentaine d'années, soit de 1955 à 1988. On peut toutefois distinguer deux périodes de rédaction : la première recouvre les années 1955 à 1965, la seconde va de 1977 à 1988. Le premier groupe relate surtout les avancées théoriques de Xenakis, en relation directe avec sa production musicale. En effet, chacune de ses premières œuvres est fondée sur de nouveaux concepts. Par ailleurs, en 1963, il publiait son premier ouvrage théorique d'envergure,
11) Le titre, choisi par l'auteur, peut se traduire par « cheminements ».

(2) La musique étant perçue comme une expérience du sensible/sensoriel, le manque d'outils conceptuels quant à l'analyse en profondeur du phénomène (comparé à la littérature et aux arts visuels), a contribué à la «ghettoïsation » de celle-ci, la reléguant trop souvent au rang de noble divertissement. De nos jours, la musicologie, de plus en plus polyvalente, s'efforce de corriger cette tendance. 
Musique formelles. Nouveaux principes formels de composition musicale ${ }^{|3|}$, suivi de Musique Architecture ${ }^{(4)}$ en 1971. Sa production ultérieure se situe dans le développement de cette première période de recherche, ce qui pourrait expliquer en partie qu'il n'ait que peu écrit par la suite. Le second groupe d'articles, publiés, pour la plupart, dans les années 1980, fait davantage état de considérations philosophiques et sociales et démontre une remarquable maturation de la pensée xenakienne.

\section{Cheminements}

Bien qu'il ne s'agisse pas chronologiquement du premier texte de Xenakis, le premier article qui nous est donné à lire, dans ce recueil, a été judicieusement choisi : "Les chemins de la composition musicale ${ }^{|5|}$ ", écrit en 1981, met en perspective la teneur des textes qui suivront. Cet article a d'abord le mérite d'exposer clairement le rapport art/science tel qu'entrevu par Xenakis et, dans un deuxième temps, de résumer les assises théoriques de son langage musical. D'emblée, Xenakis jette un regard archéologique sur l'ensemble de la production musicale ${ }^{|6|}$ à travers l'histoire, dont les matériaux forment des strates dont la surprenante diversité explique :

[...] qu'ils soient riches en créations nouvelles, mais aussi en fossilisations, en ruines et en étendues désertes; et tout cela est perpétuellement en formation et en transformation; comme les nuages, les univers musicaux sont différenciés et éphémères. [...] Quelle est l'essence de ces matériaux? C'est l'intelligence humaine dans un certain état de cristallisation. Une intelligence qui cherche, qui questionne, déduit, révèle et prévoit à tous les niveaux. II semble que la musique et les arts en général doivent nécessairement être une cristallisation, une matérialisation de cette intelligence. (1994, p. 15)

De cette façon, l'art est placé dans une perspective "évolutionniste » qui induit l'idée de progrès ${ }^{(7)}$; à cet égard, Xenakis dresse un tableau comparatif de l'évolution de la musique et des mathématiques qui tend à démontrer que la faculté d'abstraction croissante est un trait fondamental commun aux deux domaines, de même que le mode expérimental qui les régit. Si l'on admet que les mathématiques fournissent à l'homme des outils d'interprétation et de prospection de l'univers, on peut admettre en corollaire que ces mêmes outils peuvent lui servir à la construction d'« objets» qui «expriment» ses structures mentales. De plus, l'artiste devrait se doter de connaissances générales dans les divers domaines des sciences physiques et humaines, afin d'acquérir une sorte d'universalité qui soit orientée vers l'étude des formes et des architectures. D'ailleurs, Xenakis préconise la création d'une science de la
(3) Musiques formelles. Nouveaux Principes de composition musicale; la Revue Musicale, 1963, Paris, Richard Massé (épuisé). Nouvelle édition, 1981 , Paris et Londres, éd. Stock. Formalized Music (revisé el augmenté), 1971, Bloomington, Indiana University Press. Nouvelle édition lavec ajouls ef corrections de Sharon Kanach), 1991, Pendragon Press, Stuyvesant (NY). (4) Musique Architecture, Tournai (Belgique), Casterman, $2^{\mathrm{e}}$ édition revue et augmentée. 1976.
(5) "Le compositeur et l'ordinateur», IRCAM, 1981.

(6) Tous genres confondus : musique classique, contemporaine, pop, traditionnelle, etc.

(7) Idée fort discutée dans les courants de pensée "postmodernes" qui voient d'avantage l'art comme l's expression * d'une société, sans égard à une quelconque idée de progrès. 
«morphologie générale» qui traiterait de ces notions, appliquées à diverses disciplines, "dans leurs aspects invariants, ainsi que des lois qui président à leurs transformations, celles-ci ayant parfois duré plusieurs millions d'années». Dans cette perspective, l'informatique est proposée comme l'outil le plus approprié au développement de la recherche et de la connaissance.

La deuxième partie de cet article traite des méthodes de composition, qui vont des applications de procédés stochastiques ${ }^{(8)}$ à la théorie des cribles ${ }^{(9)}$ (macro-composition), de la production d'ondes périodiques - synthèse de Fourier - à la production d'ondes délibérément non périodiques - mouvement brownien - et de l'interaction des deux modèles par l'injection de procédés stochastiques (micro-composition). En résumé, Xenakis pose les termes de la composition musicale dans une dialectique - déterminisme/indéterminisme induisant la notion de modèle-entité, incluant l'idée de périodicité et leur négation par des transformations continues au moyen de procédés stochastiques ${ }^{(10)}$.

L'entropie ${ }^{(11)} d^{\prime}$ une entité croit d'un certain delta à chacune des reproductions de cette entité, c'est-à-dire que l'information la concernant se dégrade en partie à chaque renouvellement, et ceci irrémédiablement. Or, il appartient au compositeur, en se fiant à l'intuition et en recourant au raisonnement, de doser la croissance de ces deltas à tous les niveaux, macroscopique, intermédiaire et microscopique, de la composition musicale. En d'autres termes, il faut fixer l'échelle de toutes les valeurs qui séparent les deux bornes du déterminisme, qui correspond à la périodicité au sens strict, et de l'indéterminisme, qui correspond au renouvellement, c'est-à-dire à la périodicité au sens large. C'est là qu'est le véritable clavier de la composition musicale. Et c'est ainsi que nous pénétrons dans un domaine aux innombrables perspectives scientifiques et philosophiques, telles que la continuité et la discontinuité des mathématiciens et l'espacetemps de la physique quantique. (1994, p. 26-27.)

Cet article démontre une synthèse remarquable de la pensée xenakienne, qui s'est bâtie par étapes; les écrits de la première période (1955-1965) posent les principaux jalons.

"La crise de la musique sérielle ${ }^{(12)}$ 》. Paru en 1955, cet article a provoqué une polémique qui a contribué à isoler davantage Xenakis des milieux de l'avant-garde musicale. Ayant démontré que l'écriture sérielle est essentiellement contrapuntique et linéaire, il fait remarquer que la polyphonie se détruit elle-même par sa trop grande complexité et que le résultat entendu est en contradiction avec le système : "La complexité énorme empêche l'audition de suivre l'enchevêtrement des lignes et a comme effet macroscopique une dispersion irraisonnée et fortuite des sons sur toute l'étendue du spectre sonore.» (1994, p. 41-42.) En fait, ce n'est pas tant le résultat qu'il critique que la méthode. Il propose donc comme solution de casser le déterminisme
(8) Stochastique: de stóchos (but), en référence à la loi des grands nombres qui implique une évolution vers un état stable, une sorte de but (Xenakis, p. 16). (9) Construction d'échelles à partir d'éléments symétriques observés dans une série de points quelconques. (10) Xenakis s'appuie sur la dualité opposant les thèses de Parménide et d'Héraclite. Parménide : l'Être doit exister toujours et partout à l'état homogène et permanent. Héraclite : rien n'est immuable, tout change constamment. Les deux thèses semblent irréductibles à première vue, mais leur interaction peut être conceptuellement féconde, l'Être-entité s'insère dans le flux du temps qui altère ef transforme ses structures de façon continue et irréversible.

(11) «L'entropie caractérise l'élat de désordre d'un système et permet de préciser quantitativement le second principe de thermodynamique : l'entropie d'un système isolé ne peut que croître.» J.P. Sarmant, Dictionnaire de physique, Hachette.

(12) Gravesaner Blätter, n 1, 1955 
intervallique du système sériel, de le dépasser en appliquant aux sons, considérés comme éléments indépendants, la notion de probabilité. Les premières considérations théoriques de Xenakis portent essentiellement sur le traitement de l'indéterminisme.

"Lettre à Hermann Sherchen ${ }^{(13)}$ ». Dans cet article, Xenakis justifie l'emploi des calculs statistiques dans Pithoprakta, précisant qu'il ne s'agit pas là d'un pur jeu de manipulations : "La composition que j'ai écrite [Pithoprakta] existait en moi avant l'étude mathématique, qui a seulement permis une formulation plus précise, plus claire [...]. » II fait mention ici de deux traits caractéristiques de son œuvre, les glissandi (continuité) et les états massiques (discontinuité) (14). Dans ces concepts, il voit le dépassement de la dualité harmonie-contrepoint : "L'être unique derrière ces deux visages [...] pourrait être une notion de densité de fréquences qui est variable dans le temps et qui, donc, tantôt est un agrégat vertical, tantôt une suite horizontale de sons. » (1994, p. 45.)

"Théorie des probabilités et composition musicale ${ }^{(15)}$. Ce texte, paru en même temps que le précédent, a une teneur nettement plus scientifique et lui sert de prolongement théorique. Ici, Xenakis fait la démonstration, à partir de Pithoprakta, de calculs appliqués à des paramètres du son tels que la durée, la hauteur et la vitesse. La moyenne statistique des écarts entre les points jetés sur une droite et le calcul probabiliste de leur variabilité s'applique aux durées. Les hauteurs étant considérées comme des éléments ponctuels dans une durée quelconque, il s'agit, à partir d'une densité moyenne connue, de calculer la probabilité de telle ou telle autre densité au moyen de la loi de Poisson. La notion de vitesse est appliquée aux sons glissés, par analogie à la loi de Boltzman et Maxwell donnant la répartition des vitesses des molécules d'un gaz pour une température donnée, transposée à une dimension (linéaire). Ces méthodes de calculs ont amené Xenakis à une représentation graphique (sur plan cartésien) de la musique précédant la transcription en notation traditionnelle. L'intérêt de la méthode réside dans l'interaction entre ses éléments en référence aux corrélations qui existent entre les composantes du son. Mais l'intérêt supérieur de tout cela se trouve surtout dans ses conséquences esthétiques qui ont amené le compositeur à contrôler "la transformation continue de grands ensembles de sons granulaires ou continus " et à faire surgir ces impressionnantes "sonorités " dont parle Gerassimos Solomos dans l'article publié dans le présent numéro.

"Éléments sur les procédés probabilistes (stochastiques) de composition musicale ${ }^{(16)}$. Ce texte reprend en substance les critiques concernant le système sériel et approfondit les avancées théoriques du texte précédent. Toutefois, deux idées fondamentales doivent être relevées. La première, d'ordre philosophique, tient au rôle cathartique de l'art et de la musique en particulier, "vers l'exaltation totale dans laquelle l'individu se confond, en perdant sa conscience, avec une vérité immédiate, rare, énorme et parfaite. Si
(13) Gravesaner Blätter, nº, 1956.

(14) Étals massiques en référence à des phénomènes tels que les mouvements des nuages, mouvements de foule, etc.

(15) Gravesaner Blätter, nº, 1956.

(16) Claude Samuel, Panorama de l'art musical contemporain, Gallimard, 1962. 
une œuvre d'art réussit cet exploit, ne serait-ce qu'un instant, elle atteint son but. " (1994, p. 54.) La création de cette méta-musique exige le rejet de toute convention ou habitude et son renouvellement par de nouveaux concepts. $C^{\prime}$ est dans cette perspective que Xenakis justifie ici sa démarche conceptuelle. La seconde a plus de résonances théoriques. II s'agit de la notion de "conflit externe", appelée également hétéronomie ou stratégie sonore, entre deux orchestres ou interprètes opposés, qui serait géré par une "matrice de règlements " établie selon la théorie mathématique des jeux. À la fin de cet article, Xenakis expose «les cadres généraux d'une attitude artistique qui, pour la première fois, utilise les mathématiques sous trois angles fondamentaux : 1. Résumé philosophique de l'être et de son évolution; exemple : la loi de Poisson. 2. Appui qualitatif et mécanisme du logos; exemples, la théorie des ensembles, la théorie des événements en chaîne, la théorie des jeux. 3. Instrument de mensuration qui affine l'investigation ef la réalisation, la perception aussi; exemples: le calcul de l'entropie, le calcul matriciel. (1994, p. 65-66.)

"La voix de la recherche et de la question; formalisation et axiomatisation de la musique ${ }^{(17)}$, "Cribles ${ }^{(18)}$. Ces deux textes sont fondamentaux dans l'évolution de la pensée xenakienne. Pour la première fois, elle fait intervenir la notion des structures «hors-temps» et la création de modèles. Jusque-là, Xenakis manipule la matière "en-temps » qui prend forme dans un projet spécifique. Chaque œuvre est conçue comme une sculpture unique du point de vue plastique ou comme un événement unique d'un point de vue dynamique /sans "avant» ni "après»). Sa forme et sa structure sont soumises aux loi du hasard ${ }^{(19)}$, ce qui explique chez lui le développement très important des lois probabilistes. Cette notion du modèle basée sur la théorie des cribles ${ }^{(20)}$ va affermir les assises conceptuelles de la dualité - déterminisme/indéterminisme - qui sera dorénavant au cœur des préoccupations de Xenakis. De plus, l'idée d'échelles produite par des cribles induit la notion de développement mélodique qui émergera graduellement des conceptions massiques dans les œuvres plus récentes.

Les textes dont il vient d'être question et qui constituent les deux tiers du recueil, posent, bien que de façon succincte, les principaux jalons de la pensée xenakienne. Ceux qui suivent, écrits pour la plupart dans les années 1980, n'apportent pas d'idées ou de concepts vraiment nouveaux mais prolongent et amplifient, surtout au niveau philosophique, ce qui a déjà été exposé. Toutefois, il me semble opportun d'en citer quelques passages particulièrement intéressants.

"Entre Charybde et Scylla ${ }^{(21)}$ ": partant d'une vision critique de la société, «tout se passe comme si la crise économique mondiale se reflétait dans la pénurie de la création artistique, se reflétait aussi dans la pénurie des idéologies politiques et sociales. " (1994, p. 88.) Xenakis oppose la richesse, la
(17) Preuves, n 177, novembre 1965. (18) Première version publiée dans Preuves, 1965; des extraits du texte définitif ont paru dans Redécouvrir le temps, Éd. de l'Université de Bruxelles, 1988.

(19) Entendre hasard au sens scientifique du terme.

(20) « la théorie des cribles étudie les symétries internes d'une suite de points construite intuitivement, donnée par l'observation, ou fabriquée de toules pièces par des modules à répétition.» (Cribles, in 1994, p. 87.) Cette théorie s'applique aux macrostructures (échelles) et aux microstructures (synthèse) du son.

(21) Spirales, février 1981. 
variété et les contradictions de notre monde. "Nous assistons aujourd'hui, nous participons tous, à des vitesses variables et différentes, à une sorte de big bang froid de l'intelligence humaine, ce super produit cosmique. C'est dans cette perspective super vitaliste, super optimiste que nous devrions nous placer, si on voulait garder les yeux et les oreilles ouverts. Ce qui veut dire que la navigation ne peut se faire qu'à la force du cerveau, qu'à la force de la réflexion totale de l'homme. " (1994, p. 89).

"Culture et créativité »(22) : ici, Xenakis préconise la «sauvegarde des cultures » par une restructuration de l'enseignement à tous les niveaux qui passerait par la libre circulation des valeurs culturelles (scientifiques et artistiques) au moyen des développements technologiques, de l'ordinateur en particulier.

"L'univers est une spirale ${ }^{(23)}$ »: la substance de ce texte (qui clôt le volume) se trouve résumée en cette profession de foi : «... l'artiste, le penseur, l'être humain, a le besoin impérieux d'un espoir suprême : pouvoir inventer, créer, pas seulement découvrir ou dévoiler, comme dirait Heidegger. Oui, l'originalité est une nécessité absolue de survie de l'espèce humaine. J'ajouterai qu'elle est une nécessité de survie pour le cosmos.» (1994, p. 136-137.) Mentionnons au moins les titres des autres textes: "Sur le temps", "Musique et originalité », "Des univers du son», "Condition du musicien», "Pour l'innovation culturelle ${ }^{(24)}$ 》.
(22) Cultures, vol. 3, n 4 ; La Baconnière/Les Presses de l'UNESCO (1976).

(23) Nouvel Observateur, 25-31 mai 1984.

(24) Redécouvrir le temps, Éd. de I'Université de Bruxelles, 1988. Phrréatique, $n^{\circ} 28,1984$. Boris de Schloezer \& Marina Scriabine, Problèmes de la musique moderne, Éd. de Minuit, 1977. France Forum, $n^{\circ} 223$ 224, oct.-déc. 1985. Robert Badinter, Vous avez dit fascisme?, Éditions Montalba, 1984.

\section{Pour conclure: Ars philosophica}

La vision de l'art chez Xenakis relève en partie de la philosophie positiviste, qui croit foncièrement en la notion de progrès scientifique, et, d'autre part, de la philosophie humaniste de par sa vision universalisante, qui voit l'épanouissement de l'homme passer par le développement et la force de son intelligence. Toutefois, au-delà de ces catégories traditionnelles, il place l'art et la musique en particulier dans une position privilégiée, comme la quintessence des facultés d'abstraction de l'intelligence humaine, au-dessus de la pensée scientifique elle-même. De plus, l'art est investi d'un rôle salvateur, outil de transformation du monde, outil de créativité, d'avancement, d'épanouissement, en un mot, de liberté. Grâce aux immenses moyens de communication qui se développent actuellement, l'art devrait devenir le pain quotidien de l'homme de demain, un moyen privilégié d'étendre sa conscience aux dimensions de l'univers. "En effet, si l'homme, son espèce, est à l'image de l'univers, alors à son tour l'homme en vertu du principe que l'on est forcé de poser, de création à partir du néant, alors l'homme pourrait redéfinir son univers tel un environnement qu'il s'octroierait en harmonie avec son essence créatrice ${ }^{(25)}$."

(25) «Musique et originalité ». (1994, p. 111 .) 
Pour utopique et à contre-courant des tendances de la société actuelle que peut paraître cette philosophie, elle n'en reste pas moins extraordinairement stimulante et porteuse d'espoir à une époque où il est tant question du déclin rapide de notre civilisation.

\section{Ars tragica}

Un aspect qu'on ne saurait éviter de la musique de Xenakis reste l'impact sensoriel, émotionnel qu'elle a sur son auditoire. Bien que cela ne fasse pas partie de son discours, il nous en livre quelques bribes en référence à ses souvenir de résistance en Grèce. D'abord cette fascination pour les balayages lumineux de la D.C.A., puis ce récit étonnant :

Le fleuve humain scande un mot d'ordre en rythme unanime. Puis un autre mot d'ordre est lancé en tête de la manifestation et se propage jusqu'à la queve en remplacement du premier. Une onde de transition part ainsi de la tête à la queue. La clameur emplit la ville, la force inhibitrice de la voix et du rythme est culminante. C'est un événement hautement puissant et beau dans sa férocité. Puis le choc des manifestants et de l'ennemi se produit. Le rythme parfait du dernier mot d'ordre se rompt en un amas énorme de cris chaotiques qui, lui aussi, se propage à la queve. Imaginons de plus des crépitements de dizaines de mitrailleuses et les sifflements des balles qui ajoutent leurs ponctuations à ce désordre total. Puis, rapidement, la foule est dispersée, et à l'enfer sonore et visuel succède un calme étonnant, plein de désespoir, de mort et de poussière. ("Théorie des probabilités et composition musicale» in 1994, p. 58).

Xenakis, comme des millions d'autres, a été marqué par la violence du siècle, et l'idée de la mort est au centre de sa musique et de ses concepts mêmes $^{(26)}$. Dans cette dialectique d'une entité et de sa négation, dans le processus de transformations continues incluant l'idée de périodicité, cycle de morts et de renaissances et jusqu'à sa conception des structures hors-temps voulant échapper au flux irréversible. Sa musique est sous-tendue par un double système de forces: forces positives - sublimation, catharsis; forces négatives - destructions. C'est dans la tension générée par ce double système que se trouve l'essence de la Tragédie. Xenakis, et c'est ce qui le distingue par-dessus tout des compositeurs de sa génération, porte en lui et projette dans son œuvre une dimension hautement tragique qui le relie à ces lointains ancêtres, Sophocle et Eschyle.

Ces deux aspects, philosophique et tragique, fondent la personnalité exceptionnelle de Xenakis et font de lui un classique dans toute l'acception du terme.
(26) Comment ne pas l'entendre dans les vrombissements hallucinants de ses premières œuvres, dans les clameurs de Nuits, les cris de Cassandre (Oresteïa). les déferlements de feu d'Eonta, etc.

XENAKIS, I. (1981), Musiques formelles, Paris, Stock.

XENAKIS, I. (1994), Kéleüta Écrits, Paris, éd. de L'Arche, (préface de Benoît Gibson). 